\title{
A Trotter-type scheme for the generalized gradient of the optimal value function
}

\author{
Cătălin Popa \\ Facultatea de Matematică, Universitatea "Al.I.Cuza" \\ Bdul. Copou 11, 6600 Iaşi, România \\ Fax: 40-32-146330. E-mail: cpopa@uaic.ro
}

\begin{abstract}
An iterative formula for the generalized gradient of a Trotter-type approximation for the optimal value function associated with the control of a certain nonlinear parabolic system is established. This formula is useful in constructing suboptimal feedback controls.
\end{abstract}

\section{Keywords}

Trotter product formula, dynamic programming equation, parabolic variational inequality, (sub)optimal feedback control

\section{INTRODUCTION}

In this paper we shall reveal an interesting aspect of approximation of dynamic programming Hamilton-Jacobi equations via Trotter product formulas. Also, we shall explain how this aspect is reflected in constructing suboptimal feedback controls.

One of the main objectives of optimal control theory is the construction of optimal feedback controls. Simple heuristic considerations based on dynamic programming lead to feedback laws expressed by means of the optimal value function. In many significant situations, these laws can be rigorously justified (see, for example, Barbu (1984) and Popa (to appear)). However, it remains the question: how to compute (or approximate) the optimal value function in a reasonable manner (so that the feedback law should become effective)? Certainly, this function satisfies a Hamilton-Jacobi equation (called, in this context, the dynamic programming equation), but such an equation is a very complicated mathematical object. In author's opinion, the most promising way to compute solutions of Hamilton-Jacobi equations (satisfying certain initial or final conditions), is offered by a treatment of these equations by Trotter product formulas. These formulas are obtained by breaking Hamilton-Jacobi equations into two or several parts on small intervals (that is, by decomposing the associated Cauchy problem into two or several such problems). This decoupling leads to a better understanding of the making of solutions and, from a numerical viewpoint, to decentralization of calculus. Several convergent Trotter product formulas 
was proposed by V.Barbu and the author for dynamic programming equations associated with the control of parabolic variational inequalities (see Barbu $(1988,1991)$ and Popa (1991, 1995)). Numerical tests performed by V.Arnăutu and A.Niemistö (see Arnăutu (1995) and Niemistö (to appear)) show that the Trotter product formula approach is indeed an effective and realistic way in computing solutions of dynamic programming equations, and, implicitly, in constructing suboptimal feedback controls.

The generating idea of this paper consists in two simple observations. First, if we have a Trotter product formula approach to Hamilton-Jacobi equations in view, it is much easier to calculate the gradients of solutions than the solutions themselves. In other words, Trotter approximations for the gradients of solutions are much simpler than those for the solutions. On the other hand, the expression of feedback laws explicitly contains not only the solution of the dynamic programming equation but even its gradient. So, instead of computing solutions of dynamic programming equations by Trotter schemes and then differentiating them, it is preferable to directly compute gradients of solutions by schemes of the same kind.

Our aim here is to give an iterative formula for the generalized gradient of the Trotter approximation for the solution of the dynamic programming equation associated with the control of a certain nonlinear parabolic system. This formula seems to be much more effective in constructing suboptimal feedback controls. Also, it may be interpreted as a Trotter approximation for the vector variant of the conservation law equation obtained by formal differentiation of the dynamic programming equation.

\section{AN EXAMPLE}

Let us illustrate what we have asserted above by a simple example.

Consider the Hamilton-Jacobi equation of Hamiltonian mechanics corresponding to Hamiltonian $H(p, y)=\frac{1}{2}|p|^{2}+U(y)$ :

$\left\{\begin{array}{l}S_{t}(t, y)+\frac{1}{2}\left|S_{y}(t, y)\right|^{2}+U(y)=0 \text { in }[0, T] \times \mathcal{H} \\ S(0, y)=S_{0}(y)\end{array}\right.$

where $\mathcal{H}$ is the state space. Divide the interval $[0, T]$ into $N$ subintervals of the same length $\varepsilon=T / N$. Let us decouple the terms corresponding to the two kinds of energy in (1) on each subinterval by decomposing Cauchy problem (1) into two elementary Cauchy problems. One obtains the following Trotter approximation:

$\left\{\begin{array}{l}S_{t}^{\varepsilon}(t, y)+\frac{1}{2}\left|S_{y}^{\varepsilon}(t, y)\right|^{2}=0 \text { in }((i-1) \varepsilon, i \varepsilon] \times \mathcal{H} \\ S^{\varepsilon}((i-1) \varepsilon+0, y)=S^{\varepsilon}((i-1) \varepsilon, y)-\varepsilon U(y) .\end{array}\right.$

We can express the solution of Cauchy problem (2) by using the well-known Lax representation:

$$
S^{\varepsilon}(i \varepsilon, y)=\inf \left\{\frac{1}{2 \varepsilon}|z-y|^{2}+S^{\varepsilon}((i-1) \varepsilon, z)-\varepsilon U(z): z \in \mathcal{H}\right\}, i=1,2, \ldots, N
$$


Now, solving the minimization problem contained in (3), one easily finds the following alternative (but more elaborated) formula:

$$
\left\{\begin{aligned}
S^{\epsilon}(i \varepsilon, y) & =\frac{\varepsilon}{2}\left|\left(\nabla_{y} S^{e}((i-1) \varepsilon, \cdot)-\varepsilon \nabla U\right)_{\varepsilon} y\right|^{2} \\
& +S^{e}\left((i-1) \varepsilon,\left(I+\varepsilon\left(\nabla_{y} S^{e}((i-1) \varepsilon, \cdot)-\varepsilon \nabla U\right)\right)^{-1} y\right) \\
& -\varepsilon U\left(\left(I+\varepsilon\left(\nabla_{y} S^{e}((i-1) \varepsilon, \cdot)-\varepsilon \nabla U\right)\right)^{-1} y\right), i=1,2, \ldots, N \\
S^{\epsilon}(0, y) & =S_{0}(y) .
\end{aligned}\right.
$$

Next, it is not difficult to show (see Barbu and Precupanu (1986), Thm. 2.3, p.121) that the gradient of $S^{\varepsilon}$ is given by the following iterative formula:

$$
\left\{\begin{array}{l}
\nabla_{y} S^{\varepsilon}(i \varepsilon, y)=\left(\nabla_{y} S^{\varepsilon}((i-1) \varepsilon, \cdot)-\varepsilon \nabla U\right)_{\varepsilon} y, i=1,2, \ldots, N \\
\nabla_{y} S^{\varepsilon}(0, y)=\nabla S_{0}(y)
\end{array}\right.
$$

In (4), (5) (and throughout in the sequel) the symbol $\varepsilon$ subscript after an operator means passing to its Yosida approximation (for instance, $\left(\nabla S_{0}-\varepsilon \nabla U\right)_{\varepsilon}=\frac{1}{\varepsilon}\left(I-\left(I+\varepsilon\left(\nabla S_{0}-\right.\right.\right.$ $\left.\left.\varepsilon \nabla U))^{-1}\right)\right)$.

Clearly, the iterative formula (5) for the gradient of $S^{\varepsilon}$ is simpler than that for $S^{\varepsilon}$ given by (4). To make this fact even more striking, apply (4) $i$ times. One obtains

$$
\begin{aligned}
S^{c}(i \varepsilon, y) & =\frac{\varepsilon}{2}\left|P_{1} Q_{2} Q_{3} \ldots Q_{i} y\right|^{2}+\frac{\varepsilon}{2}\left|P_{2} Q_{3} \ldots Q_{i} y\right|^{2}+\cdots+\frac{\varepsilon}{2}\left|P_{i} y\right|^{2} \\
& -\varepsilon U\left(Q_{1} Q_{2} Q_{3} \ldots Q_{i} y\right)-\varepsilon U\left(Q_{2} Q_{3} \ldots Q_{i} y\right)-\cdots-\varepsilon U\left(Q_{i} y\right) \\
& +S_{0}\left(Q_{1} Q_{2} Q_{3} \ldots Q_{i} y\right),
\end{aligned}
$$

where $P_{j}, Q_{j}$ are the nonlinear operators defined by

$$
\begin{aligned}
& \left\{\begin{array}{l}
P_{j}=\left(P_{j-1}-\varepsilon \nabla U\right)_{\epsilon}, j=1,2, \ldots, N, \\
P_{0}=\nabla S_{0},
\end{array}\right. \\
& Q_{j}=\left(I+\varepsilon\left(P_{j-1}-\varepsilon \nabla U\right)\right)^{-1}, j=1,2, \ldots, N .
\end{aligned}
$$

On the other hand, the expression of the gradient of $S^{\varepsilon}$ at $i \varepsilon$ contains only operator $P_{i}$ :

$$
\nabla_{y} S^{\varepsilon}(i \varepsilon, y)=P_{i} y=\underbrace{\left(\ldots\left(\left(\nabla S_{0}-\varepsilon \nabla U\right)_{\varepsilon}-\varepsilon \nabla U\right)_{\varepsilon} \ldots-\varepsilon \nabla U\right)_{\varepsilon}}_{i \text { iterations }} y .
$$

In conclusion, from a theoretical point of view, the iterative formula (5) for the gradient of $S^{\varepsilon}$ is more attractive than that for $S^{\varepsilon}$ given by (4) at least because the final expression (9) for $\nabla_{y} S^{\varepsilon}(i \varepsilon, y)$ is much simpler than that for $S^{\varepsilon}(i \varepsilon, y)$ given by formulas (6)-(8) (it contains only $P_{i}$ ). Finally, let us point out that $\nabla_{y} S^{\varepsilon}(i \varepsilon, y)$ (given by (5)) can be interpreted as a Trotter approximation for the solution $\nabla_{y} S(t, y)$ of the vector variant of the conservation law equation obtained by formal differentiation of Hamilton-Jacobi equation (1). In other words, $\nabla_{y} S^{\varepsilon}(i \varepsilon, y)$ can be formally obtained like $S^{\varepsilon}(i \varepsilon, y)$, but by treating (this time) the conservation law equation in the same manner as above. 


\section{THE FRAMEWORK AND THE MAIN RESULT}

All the preceding considerations can be repeated with the same effect in the more complex situation of dynamic programming equations associated with the control of nonlinear parabolic systems.

Let $\mathcal{U}$ be a real Hilbert space and set $\mathcal{H}=L^{2}(\Omega)$, where $\Omega$ is an open and bounded subset of $\mathbb{R}^{n}$ having a sufficiently smooth boundary. The control system we deal with is described by the following mixed boundary value problem:

$$
\left\{\begin{array}{l}
\frac{\partial y}{\partial t}+A_{0} y+\beta(y) \ni B u \text { a.e. in }(0, T) \times \Omega \\
y=0 \text { on }(0, T) \times \partial \Omega \\
y(0, x)=y^{0}(x) \text { in } \Omega
\end{array}\right.
$$

Here $A_{0}$ is the elliptic differential operator defined by

$A_{0} y=-\sum_{i, j=1}^{n} \frac{\partial}{\partial x_{j}}\left(a_{i j}(x) \frac{\partial y}{\partial x_{i}}\right)+a_{0}(x) y$

where $a_{i j} \in C^{1}(\Omega), a_{0} \in L^{\infty}(\Omega), a_{i j}=a_{j i}, a_{0}(x) \geq 0$ a.e. $x \in \Omega$, and, for some $\omega>0$,

$\sum_{i, j=1}^{n} a_{i j}(x) \xi_{i} \xi_{j} \geq \omega \sum_{i=1}^{n}\left|\xi_{i}\right|^{2}$ for all $\left(\xi_{1}, \xi_{2}, \ldots, \xi_{n}\right) \in \mathbb{R}^{n}$, a.e. $x \in \Omega$.

The nonlinear term $\beta$ is a maximal monotone graph in $\mathbb{R}^{2}$ containing $(0,0)$. Take a convex function $j: \mathbb{R} \rightarrow(-\infty,+\infty]$ whose subdifferential is $\beta$, and define the convex function $\phi: \mathcal{H} \rightarrow(-\infty,+\infty]$ as $\phi(y)=\int_{\Omega} j(y(x)) d x$. Operator $B$ from $\mathcal{U}$ to $\mathcal{H}$ is linear and continuous, and $y^{0} \in \overline{D(\phi)}$.

A standard existence result (see Barbu (1984), Thm. 4.3, p.131) states that under the above assumptions on $A_{0}, \beta, B$ and $y^{0}$, problem (10) has a unique solution $y \in C([0, T] ; \mathcal{H})$ such that $\sqrt{t} y^{\prime} \in L^{2}(0, T ; \mathcal{H})$ and $\sqrt{t} y \in L^{2}\left(0, T ; H_{0}^{1}(\Omega) \cap H^{2}(\Omega)\right)$. Let us also mention that problem (10) can be interpreted as an evolution equation in $\mathcal{H}$, that is, the solution $y$ of (10) satisfies

$y^{\prime}+A y+\beta(y) \ni B u$ a.e. in $(0, T)$,

where $A$ is the linear continuous operator from $\mathcal{V}=H_{0}^{1}(\Omega)$ to $\mathcal{V}^{\prime}=H^{-1}(\Omega)$ defined by

$(A y, z)=\sum_{i, j=1}^{n} \int_{\Omega} a_{i j} \frac{\partial y}{\partial x_{i}} \frac{\partial z}{\partial x_{j}} d x+\int_{\Omega} a_{0} y z d x$ for all $y, z \in H_{0}^{1}(\Omega)$.

Consider the following optimal control problem:

(P) Minimize

$\int_{0}^{T}(h(u(t))+g(y(t))) d t+\ell(y(T))$

over all $u \in L^{2}(0, T ; \mathcal{U})$, where $y \in C([0, T] ; \mathcal{H})$ satisfies $(10)$.

Impose on the functions $h, g, \ell$ the following hypotheses: 
(H1) $h: \mathcal{U} \rightarrow(-\infty,+\infty]$ is convex, lower semicontinuous, not identically $+\infty$ and, for some $c_{1}>0$ and $c_{2} \in \mathbb{R}$, satisfies

$$
h(u) \geq c_{1}|u|^{2}-c_{2} \text { for all } u \in \mathcal{U} .
$$

(H2) $g, \ell: \mathcal{H} \rightarrow \mathbb{R}$ are Lipschitz continuous on bounded subsets and bounded from below by affine functions.

We associate with problem $(\mathrm{P})$ the corresponding optimal value function $V:[0, T] \times$ $\overline{D(\phi)} \rightarrow \mathbb{R}:$

$$
\begin{aligned}
V(t, y)=\inf \left\{\int_{t}^{T}(h(u(s))+g(z(s))) d s+\ell(z(T)):\right. \\
\left.z^{\prime}+A z+\beta(z) \ni B u \text { a.e. in }(t, T), z(t)=y, u \in L^{2}(t, T ; \mathcal{U})\right\} .
\end{aligned}
$$

One knows that for every optimal pair of problem (P), the following feedback law holds (see Barbu (1984), Thm. 5.6, p.208, and Popa (to appear), Thm. 2.3):

$u^{*}(t) \in \partial h^{*}\left(-B^{*} \partial_{y} V\left(t, y^{*}(t)\right)\right)$ a.e. $t \in(0, T)$.

Here $h^{*}$ is the convex conjugate of $h, B^{*}$ is the adjoint of $B$, and $\partial_{y} V(t, y)$ is the generalized gradient (in Clarke's sense) of $y \mapsto V(t, y)$.

It is also well-known that the optimal value function $V$ satisfies (in a certain generalized sense) the following Hamilton-Jacobi equation:

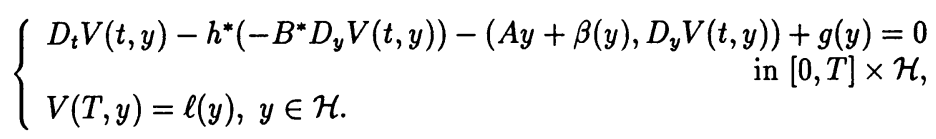

One can obtain Trotter-type approximations for $V$ by treating equation (12) in a similar manner as in the preceding section. Let $\varepsilon=T / N$. Decoupling the last three terms in the left-hand side of $(12)$ on each subinterval $[i \varepsilon,(i+1) \varepsilon]$ and then using a Lax-type representation formula, we get (in a heuristic manner) the following Trotter scheme, proposed in Popa (1995) (for simplicity, we shall indicate it only for $t=i \varepsilon$ ):

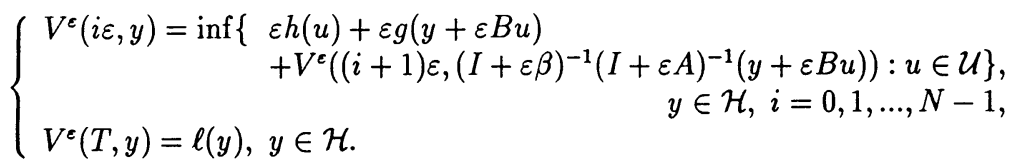

The fact that $V^{\varepsilon}$ really approximates $V$ is not trivial in the case of nonlinear and infinitedimensional control systems (see Popa (1995), Thm. 3.1).

The following question arises at this point: If we take $V^{\varepsilon}$ instead of $V$ in feedback law (11), does the new feedback law provide suboptimal controls (that is, approximately optimal controls) for problem (P)? All that we know at the moment is that the answer is positive in the following sense: Consider the following discrete approximation for $(\mathrm{P})$ (see Popa (1995), Thm. 5.1): 
$\left(\mathrm{P}^{\varepsilon}\right)$ Minimize

$\sum_{i=1}^{N} \varepsilon\left(h\left(u_{i}\right)+g\left(y_{i}\right)\right)+\ell\left(y_{N}\right)$

over all $N$-tuples $\left(u_{1}, u_{2}, \ldots, u_{N}\right) \in \mathcal{U}^{N}$, where $\left(y_{1}, y_{2}, \ldots, y_{N}\right) \in \mathcal{H}^{N}$ satisfies the scheme

$\left\{\begin{array}{l}y_{i}=(I+\varepsilon \beta)^{-1}(I+\varepsilon A)^{-1}\left(y_{i-1}+\varepsilon B u_{i}\right), i=1,2, \ldots, N, \\ y_{0}=y^{0} .\end{array}\right.$

(Note that scheme (14) can be viewed as a Trotter approximation for the state equation (10).) Then, for every optimal $N$-tuple $\left(u_{1}^{\varepsilon}, u_{2}^{\varepsilon}, \ldots, u_{N}^{\varepsilon}\right)$ of problem $\left(\mathrm{P}^{\varepsilon}\right)$, the following discrete version of feedback law (11) holds (see Popa (1995), Thm. 6.1):

$\left\{\begin{array}{l}u_{i}^{\varepsilon} \in \partial h^{*}\left(-B^{*} \partial_{y} V^{\epsilon}\left((i-1) \varepsilon, y_{i-1}^{\varepsilon}\right)\right) \\ y_{i}^{\varepsilon}=(I+\varepsilon \beta)^{-1}(I+\varepsilon A)^{-1}\left(y_{i-1}^{\varepsilon}+\varepsilon B u_{i}^{\varepsilon}\right), i=1,2, \ldots, N \\ y_{0}^{\varepsilon}=y^{0}\end{array}\right.$

It is clear (if we regard scheme (15)) that to construct suboptimal feedback controls for problem (P), we must be able to compute $\partial_{y} V^{e}(i \varepsilon, y)$. Our aim is to give an iterative formula for $\partial_{y} V^{\varepsilon}(i \varepsilon, y)$ analogous to that for $\nabla_{y} S^{\varepsilon}(i \varepsilon, y)$ established before.

The following additional hypotheses are needed:

(H3) $g, \ell$ are convex and bounded on bounded subsets.

(H4) If $y, z \in \mathcal{H}$ satisfy $y \leq z$ a.e. in $\Omega$, then $g(y) \leq g(z)$ and $\ell(y) \leq \ell(z)$.

Set $V_{-}^{\varepsilon}((i+1) \varepsilon, y)=V^{\varepsilon}\left((i+1) \varepsilon,(I+\varepsilon \beta)^{-1}(I+\varepsilon A)^{-1} y\right)+\varepsilon g(y)$. (We have already met the above expression in the definition (13) of $V^{\varepsilon}$.)

The following theorem is our main result.

Theorem 1 Under the above hypotheses on $A_{0}, \beta, B, y^{0}$ and $h$, suppose in addition that $\beta$ is concave and $g$ and $\ell$ satisfy $(H 3)$ and $(H 4)$. Then

$\partial_{y} V^{\varepsilon}(i \varepsilon, y) \subset \bigcup_{u_{i}} \partial_{y} V_{-}^{\varepsilon}\left((i+1) \varepsilon, y+\varepsilon B u_{i}\right)$,

where $u_{i}$ runs over all solutions of the inclusion

$u_{i} \in \partial h^{*}\left(-B^{*} \partial_{y} V_{-}^{\varepsilon}\left((i+1) \varepsilon, y+\varepsilon B u_{i}\right)\right), i=N-1, \ldots, 1,0$,

and

$\partial_{y} V^{\varepsilon}(N \varepsilon, y)=\partial \ell(y)$.

If $\mathcal{U}=\mathcal{H}$ and $B=I$, then

$\partial_{y} V^{\varepsilon}(i \varepsilon, y) \subset \partial_{y} V_{-}^{\varepsilon}\left((i+1) \varepsilon,\left(I-\varepsilon \partial h^{*}\left(-\partial_{y} V_{-}^{\varepsilon}((i+1) \varepsilon, \cdot)\right)\right)^{-1} y\right)$.

Sketch of the proof. First of all, let us remark that the function $y \mapsto V^{\varepsilon}(i \varepsilon, y)$ is convex on $\mathcal{H}$. (Consequently, $\partial_{y} V^{\varepsilon}(i \varepsilon, y)$ will coincide with the subdifferential of $y \mapsto V^{\varepsilon}(i \varepsilon, y)$ 
in the sense of convex analysis.) Indeed, by convexity of $\ell$ and $r \mapsto(I+\varepsilon \beta)^{-1}(r)$ (do not forget that $\beta$ is concave), using also (H4), we infer that the function $y \mapsto$ $\ell\left((I+\varepsilon \beta)^{-1}(I+\varepsilon A)^{-1} y\right)$ is convex on $\mathcal{H}$. Also, this function is nondecreasing in the sense of (H4). (It suffices to apply the monotonicity of $r \mapsto(I+\varepsilon \beta)^{-1}(r)$ and the maximum principle to the elliptic operator $I+\varepsilon A_{0}$.) Using both these properties of $y \mapsto \ell\left((I+\varepsilon \beta)^{-1}(I+\varepsilon A)^{-1} y\right)$ (and $g$ ), it is not difficult to show that $y \mapsto V^{\varepsilon}((N-1) \varepsilon, y$ ) is convex and nondecreasing (see also the proof of Lemma 4.1 in Popa (to appear)). Now we successively argue $N-i$ times as above to obtain that $y \mapsto V^{e}(i \varepsilon, y)$ is convex on $\mathcal{H}$ (and nondecreasing). Consequently, $y \mapsto V_{-}^{\varepsilon}((i+1) \varepsilon, y)$ is convex too.

Then, we interpret the minimization problem (13) defining $V^{e}$ as a discrete optimal control problem with convex performance criterion, where the state variable takes only two values: the initial value $y$ and the final one $y+\varepsilon B u$. For this problem, it is easy to derive the following discrete Pontryagin-type maximum principle (see also formulas (6.17), (6.18) in Popa (1995)): For any optimal element $u_{i} \in \mathcal{U}$, there exists $p_{i} \in \mathcal{H}$ such that

$\left\{\begin{array}{l}p_{i} \in-\partial_{y} V_{-}^{\varepsilon}\left((i+1) \varepsilon, y+\varepsilon B u_{i}\right) \\ u_{i} \in \partial h^{*}\left(B^{*} p_{i}\right) .\end{array}\right.$

(The characteristic feature of the above optimality conditions is that the discrete costate is constant, that is, the initial costate coincides with the final one.)

Since $y \mapsto V_{-}^{\varepsilon}((i+1) \varepsilon, y)$ is convex, we can use the same argument of the proof of Proposition 2.2 from Barbu and Precupanu (1986), p. 317, to prove that

$\partial_{y} V^{\varepsilon}(i \varepsilon, y)=\left\{-p_{i} \in \mathcal{H}:\right.$ there exists $u_{i} \in \mathcal{U}$ such that $\left(p_{i}, u_{i}\right)$ satisfies (19) $\}$.

But (20) in conjunction with (19) gives (16) and (17).

A result similar to the above theorem was formulated by Barbu but for convex control problems governed by linear parabolic equation (see Barbu (to appear)).

Theorem 1 says that to compute $\partial_{y} V^{\varepsilon}(i \varepsilon, y)$, we only need to know $z \mapsto \partial_{y} V_{-}^{\varepsilon}((i+1) \varepsilon, z)$. However, to express the gradient of $V_{-}^{\varepsilon}$ (with respect to $y$ ) in terms of the gradient of $V^{\varepsilon}$, we need some adequate chain rules. So, one can successively compute $\partial_{y} V^{\varepsilon}(i \varepsilon, y)$ $(i=N-1, \ldots, 1,0)$ by starting with $\partial_{y} V^{\varepsilon}(N \varepsilon, y)=\partial \ell(y)$. Here is an example.

Corollary 1 If $\beta \in C^{1}(\mathbb{R}), \mathcal{U}=\mathcal{H}$ and $B=I$, then (18) can be written in the form

$\partial_{y} V^{\varepsilon}(i \varepsilon, y) \subset-\partial h\left(-\left(-\partial h^{*}\left(-\partial_{y} V_{-}^{\varepsilon}((i+1) \varepsilon, \cdot)\right)\right)_{\varepsilon} y\right)$

where

$$
\begin{aligned}
\partial_{y} V_{-}^{\varepsilon}((i+1) \varepsilon, y)= & (I+\varepsilon A)^{-1}\left(1+\varepsilon \beta^{\prime}\left((I+\varepsilon \beta)^{-1}(I+\varepsilon A)^{-1} y\right)\right)^{-1} \\
& \partial_{y} V^{\varepsilon}\left((i+1) \varepsilon,(I+\varepsilon \beta)^{-1}(I+\varepsilon A)^{-1} y\right)+\varepsilon \partial g(y)
\end{aligned}
$$

(Here, as before, $\varepsilon$ subscript means passing to Yosida approximation.)

Proof. One easily derives (22) by using Theorem 2.3.10 from Clarke (1983). 
Let us point out that the iterative formulas (21), (22) for the generalized gradient of $V^{\varepsilon}$ (with respect to $y$ ) are a substantial generalization of the iterative formula (5) for the gradient of $S^{e}$. (We obtain a retrograde version of (5) if we take $h(\cdot)=\frac{1}{2}|\cdot|^{2}, g \equiv U$, $A \equiv 0$ and $\beta \equiv 0$ in (21), (22).)

\section{REFERENCES}

Arnăutu, V. (1995) Numerical results for a product formula approximation of HamiltonJacobi equations, International Journal of Computer Mathematics, 57, pp. 75-82.

Barbu, V. (1984) Optimal control of variational inequalities, Research Notes in Mathematics, 100, Pitman, London.

Barbu, V. (1988) Approximation of the Hamilton-Jacobi equations via Lie-Trotter product formula, Control Theory and Advanced Technology, 4, pp.189-208.

Barbu, V. (1991) The Fractional Step Method for a Nonlinear Distributed Control Problem, in Differential Equations and Control Theory (ed. V.Barbu), Pitman Research Notes in Mathematics Series, 250, Longman Scientific \& Technical, Harlow, Essex, pp. $7-16$.

Barbu, V. (to appear) Approximation of Hamilton-Jacobi Equations and Suboptimal Feedback Controllers, in Proceedings of Stefan Banach Institute Conference on Free Boundary Problems, Warsaw, November 1994.

Barbu, V. and Precupanu, Th. (1986) Convexity and optimization in Banach spaces, Second edition, Editura Academiei, Bucureşti and D. Reidel Publ. Co., Dordrecht, Boston, Lancaster.

Clarke, F.H. (1983) Optimization and nonsmooth analysis, John Wiley, New York.

Niemistö, A. (to appear) Realization of suboptimal feedback controller for evolution inclusions with numerical tests.

Popa, C. (1991) Trotter product formulae for Hamilton-Jacobi equations in infinite dimensions, Differential and Integral Equations, 4, pp. 1251-1268.

Popa, C. (1995) Feedback laws for nonlinear distributed control problems via Trottertype product formulae, SIAM Journal on Control and Optimization, 33.

Popa, C. (to appear) The relationship between the maximum principle and dynamic programming for the control of parabolic variational inequalities, submitted to SIAM Journal on Control and Optimization. 